\title{
The Evolution of Cataclysmic Variables
}

\author{
Lifang $\mathbf{L i}^{1,2}$, Fenghui Zhang ${ }^{1,2}$ and Zhanwen $\mathbf{H a n}^{1,2}$ \\ ${ }^{1}$ National Astronomical Observatories/Yunnan Observatory, CAS, P.O. Box 110, Kunming, \\ Yunnan Province, 650011, P.R. China (email: llf@ynao.ac.cn ) \\ ${ }^{2}$ Key Laboratory for the Structure and Evolution of Celestial Objects, CAS, P.O. Box 110, \\ Kunming, Yunnan Province, 650011, P.R. China
}

\begin{abstract}
Using Eggletons code the evolution of cataclysmic variables (CVs) is investigated. CVs might suffer the loss of mass and angular momentum during their evolution, we present the models of CVs with mass loss and angular momentum loss (AML) due to gravitation wave radiation (GR) and/or magnetic braking (MB). It is found that the loss of mass and angular momentum has significant influence on the evolution of CVs, and that the change of the star structure or their atmosphere properties is a possible mechanism which underlies a sudden change in the rate of AML owing to MB.
\end{abstract}

Keywords. Stars: cataclysmic variables, stars: evolution, stars: magnetic fiels, stars: mass-loss.

\section{Introduction}

CVs are close binaries in which the mass is transferred from a red dwarf to a white dwarf. The mass transfer and long-term evolution of CVs are driven by AML. The dominant AML mechanism in long orbital period CVs $\left(P_{\mathrm{Orb}} \gtrsim 3 \mathrm{hr}\right)$ is thought to be $\mathrm{MB}$ from the donor, whereas short-period CVs with $P_{\text {orb }}<3 \mathrm{hr}$ are assumed to be driven by AML associated with GR (Rappaport et al. 1983). The GR-driven shrinkage of orbit ultimately brings the secondary back to contact at $P=2 \mathrm{hr}$, at which point mass transfer regains. In this work, we would investigate the evolutionary properties of CVs.

\section{The Evolution of Cataclysmic Variables}

The evolution of CVs is investigated by using Eggletons code (Eggleton 1971). The loss of mass and AM is included in our models, the rate of AML is given by $\dot{J}=\dot{J}_{\mathrm{GR}}+\alpha \dot{J}_{\mathrm{mb}}$, where $\dot{J}_{\mathrm{GR}}$ and $\dot{J}_{\mathrm{mb}}$ are the rates of AML due to GR (Webbink 1975) and MB (Sills et al. $2000)$ respectively, and $\alpha$ is a adjustable parameter, $\alpha=1.0$ if $0.2 \leqslant M / M_{\odot} \leqslant 1.25$ and $0<\alpha<1.0$ if $M / M_{\odot}<0.2$, otherwise, $\alpha=0$. For mass loss in the evolution of $\mathrm{CVs}$, we assume that no mass is lost from CVs if $\left|\dot{M}_{2}\right|<\dot{M}_{\text {crit }}$ derived by Hameury et al. (1998). Once $\left|\dot{M}_{2}\right|>\dot{M}_{\text {crit }}$, the rate of mass loss from CVs is given by $\dot{M}=\dot{M}_{\text {crit }}+\dot{M}_{2}$. One of our evolutionary models with $\omega=15 \omega_{\odot}$ (where $\omega_{\odot}$ is the angular frequency of the Sun) is shown in Fig. 1 and in the right panel of Fig. 2. The red, green and blue lines represent the results of our model $\left(M_{2,0}=0.5 M_{\odot}, M_{\mathrm{WD}}=0.80 .5 M_{\odot}\right.$, and $\left.Z=0.02\right)$ with $\alpha=0.005, \alpha=0.01$ and $\alpha=0.015$, respectively.

\section{Discussion and Conclusions}

Most uncertainties in the theoretical investigation of CVs concern AML and the explanation of the observed period-gap as shown by the right panel of Fig. 2 based on Ritter \& Kolb (2003). A standard model (Rappaport et al. 1983) can explain the period gap of CVs. In this model, the dominant AML mechanism in long-period CVs $\left(P_{\text {orb }} \gtrsim 3 \mathrm{hr}\right)$ 

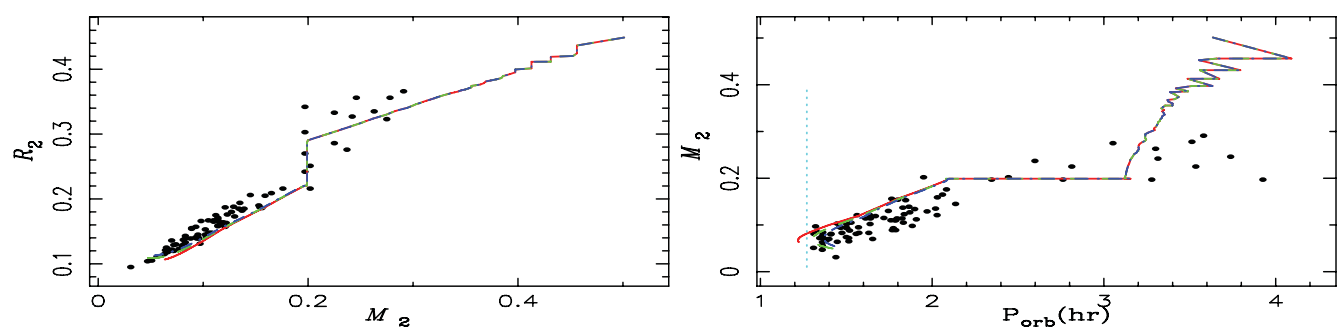

Figure 1. The left panel shows mass-radius relation of the secondary, the right panel shows the change of the secondary's mass with the orbital period of CV. The solid dots express the observations, the lines represent our model, and the dashed vertical line represents the minimum orbital period of about 72 min.
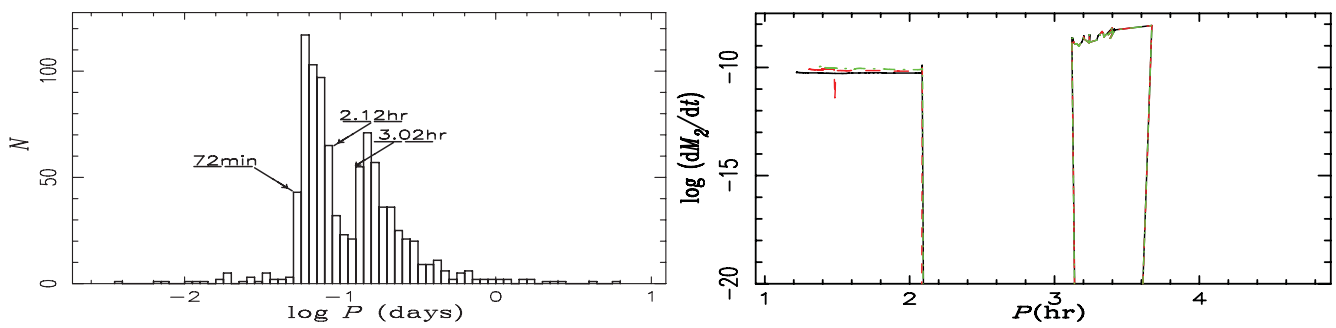

Figure 2. The left panel shows the period distribution of the observed cataclysmic variables. The right panel shows the evolution of mass loss rate of the secondary with the orbital period in our model.

is GR and MB, whereas short-period systems $\left(P_{\text {orb }}<3 \mathrm{hr}\right)$ are assumed to be driven by AML only associated with GR, i.e the MB is suddenly stopped at a orbital period $P_{\text {orb }} \approx 3 \mathrm{hr}$ when the donor in CVs becomes fully convective. The GR-driven shrinkage the orbit ultimately brings the donor back into contact at $P_{\text {orb }} \approx 2 \mathrm{hr}$, and the mass transfer regains. Hurlry et al. (2000) argued that the stars with $M \lesssim 0.35 M_{\odot}$ are fully convective. However, a sudden change in the mass-radius of the donor in the CVs occurs at $M \approx 0.2 M_{\odot}$ rather than at $M \approx 0.35 M_{\odot}$, it might be caused by a deviation from the thermal equilibrium configuration in the donors $\left(0.2 \lesssim M / M_{\odot} \lesssim 0.35\right)$ owing to its rapid mass loss, i.e. these donors in CVs are not fully convective (Knigge 2006). Meanwhile, it is seen in Fig. 1 that our model is in good agreement with the observations from knigge (2006). Additionally, no jump is observed in the measurement of surface magnetic fields of low-mass stars or even brown dwarfs, therefore this sudden change in the rate of AML due to MB is probably caused by a transition of the atmosphere of these low-mass objects to the neutral atmosphere (Mohanty \& Basri 2003). The detailed results of CVs through the population synthesis based on this work would be published elsewhere.

\section{References}

Eggleton, P. P. 1971, MNRAS, 151, 351

Hameury, J. M., Menou, K., Dubus, G., Lasota, J. P., \& Hure, J. M., 1998, MNRAS, 298, 1048

Hurley, J. R., Pols, O. R., \& Tout, C. A., 2000, MNRAS, 315, 543

Knigge, C. 2006, MNRAS, 373, 484

Mohanty, S. \& Basri, G. 2003, ApJ, 583, 451

Rappaport, S., Verbunt, F., \& Joss, P. C. 1983, ApJ, 275, 713

Ritter, H. \& Kolb, U. 2003, A\&SA, 404, 301

Sills, A., Pinsonneault, M. H., \& Terndrup, D. M., 2000, ApJ, 534, 335

Webbink, R. F. 1975, PhD Thesis (Univ. Cambridge) 